\title{
Validity of TIMI Score for Predicting 14-Day Mortality of Non-ST Elevation Myocardial Infarction Patients
}

\author{
Dileep Kumar ${ }^{1}$, Tahir Saghir ${ }^{1}$, Maham Zahid ${ }^{2}$, Arti Ashok ${ }^{1}$, Mukesh Kumar ${ }^{1}$, Arshad Ali Shah ${ }^{3}$, Izza \\ Shahid ${ }^{4}$, Sajjad Ali ${ }^{2}$, Ayema Haque ${ }^{5}$, Musa Karim ${ }^{6}$ \\ 1. Cardiology, National Institute of Cardiovascular Diseases, Karachi, PAK 2. Department of Medicine, Ziauddin \\ University, Karachi, PAK 3. Cardiology, Dow University of Health Sciences, Civil Hospital, Karachi, PAK 4. Internal \\ Medicine, Ziauddin Medical College, Karachi, PAK 5. Internal Medicine, Dow University of Health Sciences, Civil \\ Hospital, Karachi, PAK 6. Statistics, National Institute of Cardiovascular Diseases, Karachi, PAK
}

Corresponding author: Dileep Kumar, dileep_dewani2011@yahoo.com

\section{Abstract}

\section{Background}

Accurate management of non-ST elevation myocardial infarction (NSTEMI) patients can be achieved by stratifying risks as early as possible on hospital admission. Previously, the Thrombolysis in Myocardial Infarction (TIMI) risk score has been validated and used on patients presenting with NSTEMI or unstable angina (UA) in developed countries. The aim of this study was to assess the validity of the TIMI risk score in patients presenting with NSTEMI in Pakistan.

\section{Methods}

This cross-sectional study was undertaken on 300 patients who were diagnosed with NSTEMI. Data were collected from medical records, the TIMI score was calculated, and 14-day outcome was recorded. The receiver operating characteristic (ROC) curve analysis was performed, and area under the curve (AUC) along with $95 \%$ confidence interval (CI) was reported. Univariate and multivariate logistic regression analysis was performed and odds ratio (OR) along with $95 \% \mathrm{CI}$ was reported.

\section{Results}

This cross-sectional study was undertaken on 300 patients who were diagnosed with NSTEMI. Data were collected from medical records, the TIMI score was calculated, and 14-day outcome was recorded. Validity of TIMI score in predicting hospital mortality 14 days after the diagnosis of NSTEMI in a population in Pakistan was assessed by ROC curve and logistic regression analysis. The AUC of the TIMI score for predicting 14-day outcome was 0.788 [95\% CI: 0.689-0.887], with optimal cutoff of $\geqslant 4$ with sensitivity of $77.78 \%$. On multivariate analysis, cardiac arrest at presentation and the TIMI risk score were found to be independent predictors of 14-day mortality with adjusted ORs of 136.49 [10.23-1821.27] and 2.67 [1.096.57], respectively.

Review began $12 / 26 / 2020$ Review ended 01/03/2021 Published 01/06/2021

๑) Copyright 2021

Kumar et al. This is an open access article distributed under the terms of the Creative Commons Attribution License CC-BY 4.0., which permits unrestricted use, distribution, and reproduction in any medium, provided the original author and source are credited.

\section{Conclusions}

The TIMI risk score is a useful and simple score for the stratification of patients with high risk of 14-day mortality with reasonably acceptable discriminating ability in patients with NSTEMI acute coronary syndrome.

\footnotetext{
Categories: Cardiology

Keywords: non-st elevation myocardial infarction, thrombolysis in myocardial infarction, 14-day mortality, prognosis, validation
}

\section{Introduction}

Acute myocardial infarction (AMI) and unstable angina (UA) come under the broad umbrella of the term acute coronary syndrome (ACS), which accounts for nearly seven million deaths annually [1]. AMI is further classified according to ST segment deviation (ECG) which includes ST elevation myocardial infarction (STEMI) and non-ST elevation myocardial infarction (NSTEMI). Approximately 70\% of ACS patients present with NSTEMI [2], for which the treatment options are less clear [3]. Patients presenting with NSTEMI are usually older and prone to increased risk of cardiovascular complications compared to patients with STEMI [4]. Despite improvement in therapeutic interventions in the recent decade, NSTEMI still accounts for high morbidity and mortality rates [5]. This makes it crucial to use adequate risk factor assessment to determine potentially fatal cardiac complications, which may enable physicians to provide suitable and timely therapeutic management to the most vulnerable patients, thereby reducing mortality rates [6-8]. 
The Thrombolysis in Myocardial Infarction (TIMI) risk score has been demonstrated to be an effective risk stratification tool for predicting in-hospital mortality or 14-day mortality among patients with NSTEMI. Multiple trials have validated the use of the TIMI risk score in NSTEMI patients [3,9-11], which is now one of the most widely used risk stratification model for patients presenting with NSTEMI. However, the development and validation of the TIMI risk score is primarily derived from developed countries, with limited data evaluating the effectiveness of the TIMI risk score in South Asian populations despite a higher cardiovascular disease (CVD) burden [12]. This is particularly important for lower-middle-income countries like Pakistan, which are increasingly affected by CVD epidemic and encompass different genetics, lifestyle, and healthcare services compared to developed countries. Although the predictive power of the TIMI risk score has previously been validated in patients with STEMI in Pakistan [13], limited data exist regarding the validity of this risk score in patients with NSTEMI. Therefore, we sought to evaluate the validity of the TIMI risk score for predicting 14-day mortality in NSTEMI patients presenting to the largest cardiac care center of the country in an effort to determine the prognostic significance of this risk score in this cohort.

\section{Materials And Methods}

This study was conducted at a tertiary care cardiac center in Karachi during September 2019 and December 2019. After obtaining approval from the Ethics Review Committee of the institution, adult patients of either gender who presented to the Emergency Department with NSTEMI were included in the study. Informed consent was obtained from all the patients regarding their participation in the study. Patients who refused to give consent or had a history of cardiovascular surgery(s) or intervention(s) were excluded. A structured proforma was designed consisting of demographic variables, history regarding comorbidities, baseline investigations, TIMI risk score at presentation, and outcome after 14 days of admission. All patients received the ACS protocol, and management for NSTEMI was uniform for all patients as per the institutional protocol and current guidelines. For all enrolled patients, an outpatient-based follow-up visit was planned after 14 days of indexed hospitalization and telephonic calls were made for those who missed the planned follow-up visit.

Collected data were analyzed using the Statistical Package for the Social Sciences (SPSS) version 21 (IBM, Chicago, USA). The receiver operating characteristic (ROC) curve analysis was performed taking the TIMI score as the test variable and 14-day mortality as the state variable, and the area under the curve (AUC) \pm standard error (SE) along with its asymptotic $95 \%$ confidence interval (CI) were calculated. The ROC analysis was performed on R version 4.0.2 using the R-packages "ROCit," "ROCR," "dplyr," and "ggplot2." The optimal cutoff point was computed using the Youden Index method and patients were categorized into highand low-risk groups based on the computed optimal cutoff point and its association to the 14-day outcome rates was assessed using the Chi-square test. The association between survival status after 14 days and demographic and clinical characteristics of the patients were evaluated by performing appropriate Chisquare test or Student's t-test/Mann-Whitney U test. The univariate and multivariate binary logistic regression analysis were also performed to assess the strength of associations and odds ratios (ORs) and its $95 \%$ CIs were computed. Throughout the analysis $p \leqslant 0.05$ was taken as the significance criteria.

\section{Results}

A total of 300 patients with NSTEMI were included in this study. Of those, $76.7 \%$ were males while $23.3 \%$ were females. Overall, the mean age of the patients was $58.04 \pm 10.71$ years, with $21.7 \%(n=65)$ of the patients aged 65 years and above. The overall mortality rate after 14 days of procedure was $6.0 \%(n=18)$, which was significantly higher in patients with diabetes $(10.3 \%$ [13/126] vs. $2.9 \%[5 / 174] ; \mathrm{p}=0.007)$. Similarly, the 14-day mortality rate was significantly higher among patients with cardiac arrest $(90.9 \%$ $(10 / 11)$ vs. $2.8 \%(8 / 289) ; p<0.001)$ compared to those without cardiac arrest. The 14-day mortality was found to be associated with Killip class and mean heart rate at presentation, which was significantly higher in patients who did not survive $(84.33 \pm 18.09$ vs. $76.99 \pm 13.94 ; p=0.034)$. Additionally, the TIMI score was also associated with increased mortality, with a higher risk score observed in patients who did not survive compared to those who did $(4.06 \pm 0.73$ vs. $3.12 \pm 0.83 ; \mathrm{p}<0.001)$. The baseline clinical characteristics and TIMI score at presentation stratified by 14-day outcome are presented in Table 1 . 


\section{Cureus}

\begin{tabular}{|c|c|c|c|c|}
\hline \multirow{2}{*}{ Characteristics } & \multirow{2}{*}{ Total } & \multicolumn{2}{|c|}{ Outcome at 14 days } & \multirow{2}{*}{ p-value } \\
\hline & & Survived & Expired & \\
\hline $\mathrm{N}$ & 300 & 282 & 18 & - \\
\hline \multicolumn{5}{|l|}{ Gender } \\
\hline Male & $230(76.7 \%)$ & $218(77.3 \%)$ & $12(66.7 \%)$ & \multirow{2}{*}{0.301} \\
\hline Female & 70 (23.3\%) & $64(22.7 \%)$ & 6 (33.3\%) & \\
\hline Age (years) & $58.04 \pm 10.71$ & $57.77 \pm 10.41$ & $62.33 \pm 14.31$ & 0.080 \\
\hline$\leq 50$ years & $80(26.7 \%)$ & $75(26.6 \%)$ & $5(27.8 \%)$ & \multirow{3}{*}{0.144} \\
\hline $51-65$ years & $155(51.7 \%)$ & $149(52.8 \%)$ & $6(33.3 \%)$ & \\
\hline$>65$ years & $65(21.7 \%)$ & $58(20.6 \%)$ & $7(38.9 \%)$ & \\
\hline \multicolumn{5}{|l|}{ Risk factors } \\
\hline Hypertension & $253(84.3 \%)$ & $236(83.7 \%)$ & $17(94.4 \%)$ & 0.224 \\
\hline Diabetes & $126(42 \%)$ & $113(40.1 \%)$ & $13(72.2 \%)$ & $0.007^{\star}$ \\
\hline Family history of CAD & 33 (11\%) & $30(10.6 \%)$ & $3(16.7 \%)$ & 0.428 \\
\hline Smoking & $82(27.3 \%)$ & $78(27.7 \%)$ & $4(22.2 \%)$ & 0.616 \\
\hline Obesity & $27(9 \%)$ & $25(8.9 \%)$ & $2(11.1 \%)$ & 0.747 \\
\hline Dyslipidemia & $46(15.3 \%)$ & 44 (15.6\%) & $2(11.1 \%)$ & 0.608 \\
\hline Sedentary lifestyle & $93(31 \%)$ & $87(30.9 \%)$ & $6(33.3 \%)$ & 0.825 \\
\hline \multicolumn{5}{|l|}{ Killip class } \\
\hline 1 & $253(84.3 \%)$ & $250(88.7 \%)$ & $3(16.7 \%)$ & \multirow{4}{*}{$<0.001^{\star}$} \\
\hline II & $39(13 \%)$ & $29(10.3 \%)$ & $10(55.6 \%)$ & \\
\hline III & $6(2 \%)$ & $2(0.7 \%)$ & $4(22.2 \%)$ & \\
\hline IV & $2(0.7 \%)$ & $1(0.4 \%)$ & $1(5.6 \%)$ & \\
\hline Cardiac arrest & $11(3.7 \%)$ & $1(0.4 \%)$ & $10(55.6 \%)$ & $<0.001^{*}$ \\
\hline Heart rate (bpm) & $77.43 \pm 14.29$ & $76.99 \pm 13.94$ & $84.33 \pm 18.09$ & $0.034^{x}$ \\
\hline SBP (mmHg) & $120.99 \pm 17.21$ & $120.82 \pm 16.97$ & $123.72 \pm 20.99$ & 0.488 \\
\hline Serum creatinine (ng/dL) & $1.45 \pm 6.75$ & $1.44 \pm 6.96$ & $1.61 \pm 0.77$ & 0.922 \\
\hline Troponin I (ng/dL) & $7.12 \pm 17.05$ & $6.9 \pm 17.31$ & $10.48 \pm 12.3$ & 0.389 \\
\hline TIMI score & $3.18 \pm 0.85$ & $3.12 \pm 0.83$ & $4.06 \pm 0.73$ & $<0.001^{x}$ \\
\hline
\end{tabular}

\section{TABLE 1: Clinical characteristics and TIMI score at presentation stratified by 14-day outcome}

CAD, coronary artery diseases; SBP, systolic blood pressure; TIMI, thrombolysis in myocardial infarction

*significant

The ROC curve and mortality rate with respect to the TIMI score are presented in Figure 1. The predictive value, AUC of TIMI score, for predicting 14-day outcome was found to be 0.788 (95\% CI: 0.689-0.887), with optimal cutoff of $\geqslant 4$ with sensitivity of $77.78 \%$ (95\% CI: 52.36\%-93.59\%) and specificity of $68.09 \%$ (95\% CI: $62.30 \%-73.49 \%$ ). The mortality rate was significantly higher in patients with TIMI score of $\geqslant 4$ compared to those with TIMI score $<4$, with 14 -day mortality rates of $13.5 \%(14 / 104)$ versus $2.0 \%(4 / 196)(\mathrm{p}<0.001)$, respectively. 


\section{Cureus}
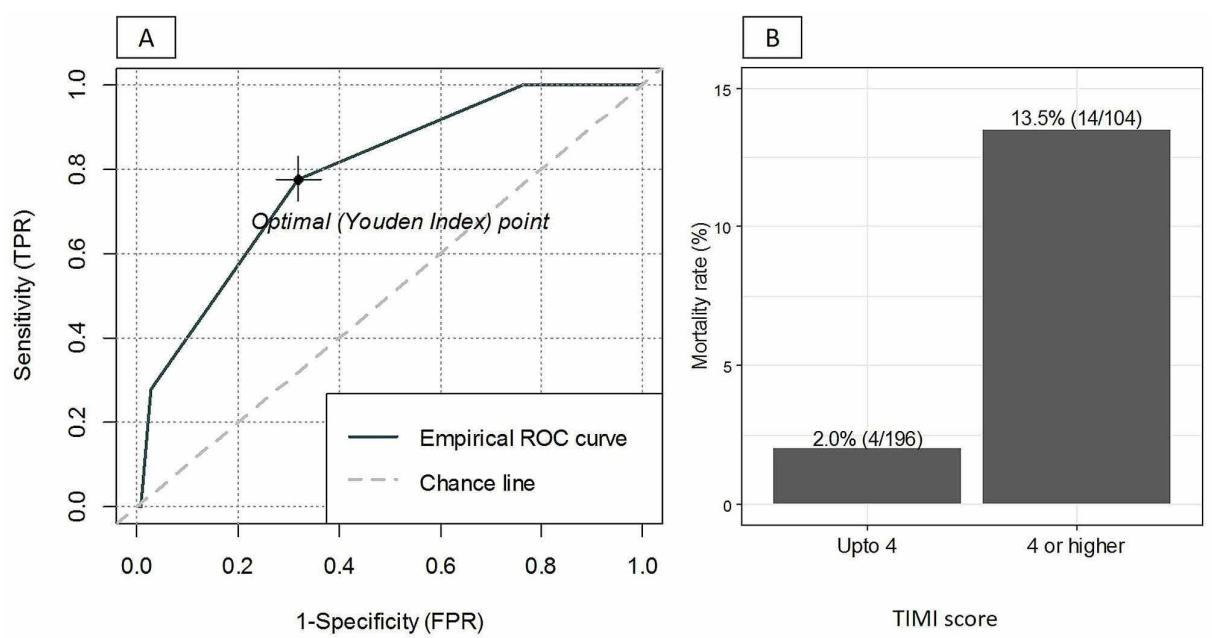

FIGURE 1: ROC curve (A) and mortality rate with respect to the TIMI score (B).

ROC, receiver operating characteristic; TIMI, thrombolysis in myocardial infarction

The univariate and multivariate logistic regression analysis for 14-day outcome are presented in Table 2. The univariate analysis revealed that heart rate (per 5 bpm increase), cardiac arrest, Killip class III or IV, and/or diabetes as comorbidity upon presentation are the key factors associated with significant increased risk of 14-day mortality with ORs of 1.16 [1.01-1.33], 351.25 [40.01-3,083.84], 35.77 [7.7-166.11], and 3.89 [1.3511.21], respectively. On multivariate analysis, cardiac arrest at presentation and the TIMI risk score were found to be independent predictors of 14-day mortality with adjusted ORs of 136.49 [10.23-1821.27] and 2.67 [1.09-6.57], respectively. 


\begin{tabular}{|c|c|c|c|c|}
\hline \multirow{2}{*}{ Characteristics } & \multicolumn{2}{|l|}{ Univariate } & \multicolumn{2}{|l|}{ Multivariate } \\
\hline & OR $[95 \% \mathrm{Cl}]$ & p-value & OR $[95 \% \mathrm{Cl}]$ & p-value \\
\hline Female & $1.7[0.61-4.72]$ & 0.306 & $2.59[0.43-15.58]$ & 0.298 \\
\hline$>65$ years & $2.06[0.78-5.4]$ & 0.143 & $1.03[0.21-5.1]$ & 0.972 \\
\hline Heart rate (per 5 bpm) & $1.16[1.01-1.33]$ & $0.038^{*}>>>>0$ & 0.94 [0.67-1.32] & 0.739 \\
\hline Serum creatinine (ng/dL) & $1[0.94-1.07]$ & 0.925 & $1.02[0.94-1.11]$ & 0.616 \\
\hline Troponin I (ng/dL) & 1.01 [0.99-1.02] & 0.427 & $1.01[0.99-1.03]$ & 0.531 \\
\hline Cardiac arrest & 351.25 [40.01-3083.84] & $<0.001^{*}$ & $136.49[10.23-1,821.27]$ & $<0.001^{*}$ \\
\hline Killip class II-IV & 35.77 [7.7-166.11] & $<0.001^{*}$ & $12.16[0.19-772.82]$ & 0.238 \\
\hline Hypertension & $3.31[0.43-25.52]$ & 0.25 & $10.31[0.03-3,505.28]$ & 0.433 \\
\hline Diabetes & 3.89 [1.35-11.21] & $0.012^{\star}$ & $2.17[0.38-12.36]$ & 0.382 \\
\hline Family history of CAD & $1.68[0.46-6.14]$ & 0.433 & $5.6[0.83-37.67]$ & 0.076 \\
\hline Smoking & $0.75[0.24-2.34]$ & 0.617 & $0.6[0.06-5.91]$ & 0.665 \\
\hline Obesity & $1.28[0.28-5.91]$ & 0.747 & 7.27 [0.88-59.75] & 0.065 \\
\hline Dyslipidemia & $0.68[0.15-3.04]$ & 0.61 & $0.13[0.01-1.7]$ & 0.118 \\
\hline Sedentary lifestyle & 1.12 [0.41-3.08] & 0.825 & $0.6[0.1-3.68]$ & 0.585 \\
\hline TIMI score & $3.32[1.83-6.02]$ & $<0.001^{*}$ & $2.67[1.09-6.57]$ & $0.032^{\star}$ \\
\hline
\end{tabular}

\section{TABLE 2: Univariate and multivariate logistic regression analysis for 14-day outcome}

CAD, coronary artery diseases; $\mathrm{Cl}$, confidence interval; OR, odds ratio; SBP, systolic blood pressure; TIMI, thrombolysis in myocardial infarction

*significant

\section{Discussion}

Patients with NSTEMI are likely to be of older age and present with multiple cardiovascular risk factors and are more prone to increased risk of cardiovascular complications. Hence, risk stratification modalities, such as the TIMI risk score, were found to be useful in stratification of high-risk patients. Hence, this study was conducted to assess the validity of the TIMI risk score for predicting 14-day mortality in NSTEMI patients in Pakistan. This study is the first of its kind in a population in Pakistan. We observed a reasonably acceptable prognostic discriminating ability of the TIMI risk score with an AUC of 0.788 (0.689-0.887), and the derived optimal cutoff of $\geqslant 4$ had a moderate sensitivity of $77.78 \%(52.36 \%-93.59 \%)$ and a specificity of $68.09 \%$ (62.30\%-73.49\%), with 14 -day mortality rates of $13.5 \%$ versus $2.0 \%(\mathrm{p}<0.001)$ for patients with TIMI scores of $\geqslant 4$ and $<4$, respectively.

The study by Antman et al. [3] proposed the TIMI risk score for NSTEMI-ACS based on seven independent predictor variables. The proposed score and event rate after 14 days of revascularization (including recurrent MI, re-revascularization, and death) were found to have significant association with monotonically increasing event rate along the TIMI score in validation cohort. The AUC value of 0.788 in our study is consistent with the C-statistics of 0.74 reported for all-cause mortality in the parent study. The TIMI score was also validated using PRISM-PLUS database by Morrow et al. [14], with a similar observation of increasing event rate ranging from $7.7 \%$ at TIMI ( 1 or 2 ) to $30.5 \%$ at TIMI (6 or 7 ). Validation and subsequent adoption of this risk score in clinical routine can be particularly useful for a country like Pakistan, where every one in four middle-aged adult presents with coronary artery disease [15].

Our analysis also demonstrates that Killip class II-IV, increased heart rate, cardiac arrest upon presentation, and history of diabetes to be significantly associated with increased risk of 14-day mortality. With respect to univariate analysis, prior observations similarly indicate Killip class and diabetes to be associated with increased 14-day mortality [16]. Diabetes is one of the most prevalent non-communicable diseases in Pakistan, with up to $11.7 \%$ of the adult population living with it, thereby ensuring the importance of the TIMI risk score as a useful predictive tool in this population cohort [17]. In developing countries like Pakistan, our results may aid physicians in evaluating their patients using a cost-effective risk stratification 
tool; hence, TIMI can be used effectively for majority population in a publicly funded healthcare setup where limited funds are available [18].

Although maximum effort was undertaken to reduce bias, there are a few that should not be overlooked. First, our study was conducted in only one tertiary cardiac care center of Pakistan, which makes it difficult to generalize the results in other cardiac centers with different populations. To accurately evaluate the validity of the TIMI risk score, large sample size from several settings should be considered. Second, we did not take medications or prescribed therapeutic interventions into consideration, which may have influenced the prognosis of these patients. Further studies should be conducted to eliminate these limitations and provide further insight into the accuracy of TIMI risk score in patients with NSTEMI in Pakistan.

\section{Conclusions}

The TIMI risk score is a useful and simple score for the stratification of patients with high risk of 14-day mortality with reasonably acceptable discriminating ability in patients with NSTEMI-ACS. Therefore, its use among clinicians is suggested to apply strategic and precise interventions in monitoring these patients. If used appropriately, this tool may prove to be a life-saving and cost-effective risk stratification tool in cardiac care settings.

\section{Additional Information \\ Disclosures}

Human subjects: Consent was obtained by all participants in this study. National Institute of Cardiovascular Diseases (NICVD), Karachi, Pakistan issued approval ERC-43/2019. Animal subjects: All authors have confirmed that this study did not involve animal subjects or tissue. Conflicts of interest: In compliance with the ICMJE uniform disclosure form, all authors declare the following: Payment/services info: All authors have declared that no financial support was received from any organization for the submitted work. Financial relationships: All authors have declared that they have no financial relationships at present or within the previous three years with any organizations that might have an interest in the submitted work. Other relationships: All authors have declared that there are no other relationships or activities that could appear to have influenced the submitted work.

\section{Acknowledgements}

The authors wish to acknowledge the support of the staff members of the Clinical Research Department of the National Institute of Cardiovascular Diseases (NICVD) in Karachi, Pakistan.

\section{References}

1. Vedanthan R, Seligman B, Fuster V: Global perspective on acute coronary syndrome: a burden on the young and poor. Circ Res. 2014, 114:1959-75. 10.1161/CIRCRESAHA.114.302782

2. Amsterdam EA, Wenger NK, Brindis RG, et al.: 2014 AHA/ACC guideline for the management of patients with non-ST-elevation acute coronary syndromes: a report of the American College of Cardiology/American Heart Association Task Force on Practice Guidelines. J Am Coll Cardiol. 2014, 64:139-228. 10.1016/j.jacc.2014.09.017

3. Antman EM, Cohen M, Bernink PJ, et al.: The TIMI risk score for unstable angina/non-ST elevation MI: a method for prognostication and therapeutic decision making. JAMA. 2000, 284:835-42. 10.1001/jama.284.7.835

4. Acharya D: Predictors of outcomes in myocardial infarction and cardiogenic shock. Cardiol Rev. 2018, 26:255-66. 10.1097/CRD.0000000000000190

5. Murray CJ, Lopez AD: Mortality by cause for eight regions of the world: Global Burden of Disease Study . Lancet. 1997, 349:1269-76. 10.1016/S0140-6736(96)07493-4

6. Roffi M, Patrono C, Collet JP, et al.: 2015 ESC Guidelines for the management of acute coronary syndromes in patients presenting without persistent ST-segment elevation. Task Force for the Management of Acute Coronary Syndromes in Patients Presenting without Persistent ST-Segment Elevation of the European Society of Cardiology (ESC). Eur Heart J. 2016, 37:267-315. 10.1714/2464.25804

7. ul Haq MA, Rudd N, Mian M, Castles A, Mogilevski T, Mutha V, Van Gaal WJ: Predictors and outcomes of early coronary angiography in patients with prior coronary artery bypass surgery presenting with non-ST elevation myocardial infarction. Open Heart. 2014, 1:000059. 10.1136/openhrt-2014-000059

8. Shaikh MK, Hanif B, Shaikh K, Khan W, Parkash J: Validation of Grace Risk Score in predicting in-hospital mortality in patients with non ST-elevation myocardial infarction and unstable angina. J Pak Med Assoc. 2014, 64:807-11.

9. Pollack Jr CV, Sites FD, Shofer FS, Sease KL, Hollander JE: Application of the TIMI risk score for unstable angina and non-ST elevation acute coronary syndrome to an unselected emergency department chest pain population. Acad Emerg Med. 2006, 13:13-8. 10.1197/j.aem.2005.06.031

10. Chase M, Robey JL, Zogby KE, Sease KL, Shofer FS, Hollander JE: Prospective validation of the Thrombolysis in Myocardial Infarction Risk Score in the emergency department chest pain population. Ann Emerg Med. 2006, 48:252-9. 10.1016/j.annemergmed.2006.01.032

11. Scirica BM, Cannon CP, Antman EM, et al.: Validation of the Thrombolysis In Myocardial Infarction (TIMI) risk score for unstable angina pectoris and non-ST-elevation myocardial infarction in the TIMI III registry. Am J Cardiol. 2002, 90:303-5. 10.1016/s0002-9149(02)02468-2 


\section{Cureus}

12. Nishtar S: Prevention of coronary heart disease in south Asia . Lancet. 2002, 360:1015-8. 10.7883/yoken1952.29.187

13. Furnaz S, Karim M, Ashraf T, et al.: Performance of the TIMI risk score in predicting mortality after primary percutaneous coronary intervention in elderly women: results from a developing country. PLoS One. 2019, 14:0220289. 10.1371/journal.pone.0220289

14. Morrow D, Antman E, Snapinn S, McCabe C, Theroux P, Braunwald E: An integrated clinical approach to predicting the benefit of tirofiban in non-ST elevation acute coronary syndromes. Application of the TIMI Risk Score for UA/NSTEMI in PRISM-PLUS. Eur Heart J. 2002, 23:223-9. 10.1053/euhj.2001.2738

15. Jafar TH, Jafary FH, Jessani S, Chaturvedi N: Heart disease epidemic in Pakistan: women and men at equal risk. Am Heart J. 2005, 150:221-6. 10.1016/j.ahj.2004.09.025

16. Kim HK, Jeong MH, Ahn Y, et al.: A new risk score system for the assessment of clinical outcomes in patients with non-ST-segment elevation myocardial infarction. Int J Cardiol. 2010, 145:450-4. 10.1016/j.ijcard.2009.06.001

17. Meo SA, Zia I, Bukhari IA, Arain SA: Type 2 diabetes mellitus in Pakistan: current prevalence and future forecast. J Pak Med Assoc. 2016, 66:1637-42.

18. Kurji Z, Premani ZS, Mithani Y: Analysis of the health care system of Pakistan: lessons learnt and way forward. J Ayub Med Coll Abbottabad. 2016, 28:601-4. 